\title{
Les émotions collectives et le mouvement des
} fédérations (1789-1790)

Collective emotions and Federation movement (1789 - 1790)

\section{Micah Alpaugh}

Traducteur : Julien Louvrier

\section{OpenEdition Journals}

\section{Édition électronique}

URL : https://journals.openedition.org/ahrf/12772

DOI : 10.4000/ahrf.12772

ISSN : 1952-403X

\section{Éditeur :}

Armand Colin, Société des études robespierristes

\section{Édition imprimée}

Date de publication : 1 juin 2013

Pagination : 49-80

ISBN : 978-2-9083-2789-2

ISSN : 0003-4436

\section{Référence électronique}

Micah Alpaugh, "Les émotions collectives et le mouvement des fédérations (1789-1790) », Annales historiques de la Révolution française [En ligne], 372 I avril-juin 2013, mis en ligne le 01 juin 2016, consulté le 01 juillet 2021. URL : http://journals.openedition.org/ahrf/12772 ; DOI : https://doi.org/ 10.4000/ahrf.12772 


\title{
LES ÉMOTIONS COLLECTIVES ET LE MOUVEMENT DES FÉDÉRATIONS (1789-1790)
}

Micah ALPAUGH

\begin{abstract}
Entre l'été 1789 et l'été 1790 , le mouvement fédératif s'étend à tout le territoire français : après des débuts modestes sous la forme de rassemblements locaux en soutien aux gardes nationaux dans la région de la Loire, il rassemble rapidement des communautés et des régions entières, jusqu'à attirer des dizaines puis des centaines de milliers de participants. Cet article revient sur l'histoire du mouvement en portant une attention particulière au rôle des émotions collectives dans la mobilisation populaire. Les récits des acteurs et témoins des fêtes fédératives, que diffusent à travers le pays les brochures, feuilles volantes, journaux et la correspondance, évoquent tous la genèse d'un nouvel esprit révolutionnaire mêlant union fraternelle et solidarité contre les ennemis de la Révolution et contribuent à attirer des foules toujours plus nombreuses. Alors que l'étude des émotions s'est très souvent limitée à l'analyse de leurs expressions littéraires et de leurs effets sur les élites, ce projet élargit quant à lui le champ d'application du concept et cherche à comprendre comment ces rassemblements pleins d'émotion ont façonné la première année de la Révolution.
\end{abstract}

Mots-clés : fédération, émotions, Garde nationale, peuple, presse, Assemblée nationale, fête, fraternité.

Le 13 juin 1790, plus de 75000 personnes convergent à Strasbourg, en bordure du Rhin, sur le Champ de la Confédération nouvellement baptisé, à l'occasion du dernier rassemblement d'une série de fédérations régionales. Ces manifestations destinées à l'origine à soutenir les bataillons locaux de la Garde nationale au cours de l'été 1789, ont attiré dès l'année suivante des dizaines voire des centaines de milliers de participants dans tout le pays. Ceux qui s'y pressent viennent d'abord y affirmer un engagement 
révolutionnaire commun, mais, à en croire les témoignages, on s'y rend aussi pour faire l'expérience de la ferveur émotionnelle qui accompagne les cérémonies. De ce point de vue, la fédération de Strasbourg tient ses promesses. La musique d'ouverture et les salves de canons provoquent chez les participants une « explosion du sentiment »; à l'issue d'une messe en l'honneur de l'Être Suprême, la prestation de serment à la Révolution et à la future Constitution déclenche une seconde vague d'acclamations, les canons étant désormais secondés par les cloches des églises de la ville. À l'expérience sonore s'ajoute le spectacle visuel : la majorité de la population locale se trouve rassemblée sur un champ immense d'où elle peut voir flotter au loin des étendards tricolores opportunément installés en haut de la pointe immense de la cathédrale de Strasbourg « pour rendre les princes jaloux [du] bonheur [du peuple français] » dans les territoires des Habsbourg sur la rive opposée. En suivant une injonction protestante strasbourgeoise à « chérir [ses] compatriotes et voisins et ne combattre que les traîtres et les tyrans », la fédération se prolonge sans troubles par une soirée de festivités et d'illuminations ${ }^{1}$. Les rassemblements et les performances collectives sont le moteur de la ferveur révolutionnaire.

L'étude des émotions et de leurs effets sur des processus politiques de grande ampleur comme la Révolution française, a fait l'objet ces dernières années d'un investissement croissant de la part des historiens. En quête de nouvelles interprétations pour expliquer le cours complexe de la Révolution, des chercheurs comme Lynn Hunt, William Reddy, Timothy Tackett, Marisa Linton et Sophie Wahnich ont attiré l'attention sur les limites de la stricte rationalité pour expliquer les actions des révolutionnaires et souligné la complexité de leur culture et de leurs expériences accumulées ${ }^{2}$. Toutefois, là où Hunt et Reddy se limitent à l'intersection des discours littéraires et

(1) Affiches des évêchés de Lorraine, 1er juillet 1790 ; Procès verbal de la fédération du Rhin (S.1, s.d.), p.15 ; « Extrait du discours prononcé en allemand par M.Blessig, Docteur en théologie de l'Université protestante, et Notable de la commune de Strasbourg », Procès-verbal de la fédération, p.70.

(2) Lynn Hunt, The Family Romance of the French Revolution, University of California Press, Berkeley, CA ,1992 ; William REDDY, The Navigation of Feeling : A Framework for the History of Emotions, Cambridge University Press, Cambridge, 2002 ; Timothy TACKETT, Par la volonté du peuple. Comment les députés de 1789 sont devenus révolutionnaires, Paris, Albin Michel, 1997 et « Conspiracy Obsession in a Time of Revolution: French Elites and the Origins of the Terror, 1789-1792», American Historical Review 105, n 3 (Juin 2000), p. 691-713; Marisa LINTON, « Fatal Friendships: The Politics of Jacobin Friendship », French Historical Studies 31, n 1 (hiver 2008), p. 51-76, et «"Do You Believe That We're Conspirators ?" : Conspiracies Real and Imagined in Jacobin Politics, 1793-1794 », dans Peter R. CAMPBELL, Thomas E. KAISER and Marisa LINTON (dir.), Conspiracy in the French Revolution, Manchester, Manchester University Press, 2007, p. 127-49 ; Sophie WAHNICH, La liberté ou la mort: essai sur la terreur et le terrorisme, Paris, La Fabrique, 2003 et La longue patience du peuple : 1792, naissance de la République, Paris, Payot, 2008. 
de la sphère publique, là où Tackett et Linton s'intéressent essentiellement aux mondes sociaux des élites politiques, et tandis que Wahnich limite son propos à la naissance de la Terreur, notre étude entend appliquer le paradigme au plus grand mouvement de rassemblements de masse pendant la Révolution, à savoir les fédérations de 1789-1790, et aux démonstrations puissantes d'émotions collectives auxquelles ces manifestations ont donné lieu.

Même si nous ne saurons jamais précisément ce que les millions de participants et d'observateurs des fédérations ont ressenti, les récits de l'époque contiennent un certain nombre de descriptions substantielles des émotions (comprises ici comme démonstrations extériorisées de sentiments) qui se sont manifestées à l'occasion de ces événements. Poussés par la nécessité d'expliquer à leurs compatriotes à la fois les rituels en formation et l'esprit même du mouvement, ces partisans ont pris la peine de détailler avec une précision inédite l'ampleur des émotions collectives engendrées par ces cérémonies. La lecture des témoignages - publics ou privés, imprimés ou manuscrits - révèle l'intérêt croissant des premiers révolutionnaires français pour les liens collectifs : il s'agit en partie de tenir en échec leurs opposants, mais également de créer les conditions d'une affirmation collective de la nouvelle ère fraternelle que la Révolution incarne pour beaucoup d'entre eux. L'importance des émotions tient essentiellement dans leur capacité à se diffuser rapidement. Le succès du mouvement des fédérations s'est construit à la fois en générant des émotions collectives et en parvenant à donner aux récits de ces fêtes une large publicité à même d'attirer toujours plus de participants et élargir ainsi la participation révolutionnaire.

Notre point de vue se démarque considérablement de l'orthodoxie historiographique sur les fédérations. Dans son livre consacré à La Fête révolutionnaire, Mona Ozouf s'intéresse à celles-ci avant tout comme création des élites révolutionnaires parisiennes et ne consacre que trois pages au mouvement fédératif largement spontané qui précède le 14 juillet $1790^{3}$. S'il est vrai que les fêtes révolutionnaires ont fait l'objet d'une prise en main croissante par les élites au fur et à mesure que la révolution progressait, ce ne fut pas le cas pour le mouvement des fédérations dans lequel les volontaires provinciaux, les communautés locales, ainsi qu'une presse périodique en plein essor ont créé et promu ensemble un modèle de participation proprement fédéral visant à inclure la nation 
française tout entière. Plus généralement, depuis le livre d'Henri Leclerq en 1929, aucune étude n'a proposé d'explorer en détail les aléas du mouvement ${ }^{4}$. En s'appuyant sur le travail de Marcel David sur le rôle de la fraternité dans la culture révolutionnaire et l'affirmation par Haim Burstin de l'importance de la participation populaire dans la construction des pratiques révolutionnaires, il y aurait beaucoup à apprendre également d'un réexamen des analyses de Jules Michelet quant à la centralité de la sociabilité et des « grands mouvements sociaux » dans la construction du nouvel ordre social à travers la France en $1789-1790^{5}$. En lieu et place d'une culture d'État dominante qui dicte les rassemblements, on y verrait que se développent rapidement des réseaux de mobilisation largement organiques, tant au niveau local que régional.

Les travaux consacrés aux mouvements sociaux ont toujours eu des difficultés à saisir le rôle des émotions collectives dans la fabrication des mouvements collectifs. Dans La psychologie des foules, un des textes fondateurs de la sociologie moderne, Gustave Le Bon considère les actions collectives comme une manifestation de «l'inconscient collectif» : les individus qui s'insèrent dans une foule perdent instantanément toute retenue et tout contrôle personnel sur leurs émotions et leurs actions ${ }^{6}$. Le paradigme opposé, développé par George Rudé et Charles Tilly, se concentre plutôt sur la rationalité des exigences des participants, soulignant particulièrement les origines concrètes des protestations et les stratégies pour établir des objectifs apparents ${ }^{7}$. De plus en plus cependant, les limites du paradigme du « choix rationnel » se sont fait jour : comment peut-on expliquer les « moments de folie » qui surviennent dans les actions collectives ou encore les émotions que les grands rassemblements peuvent produire ${ }^{8}$ ? Même dans les actions

(4) Henri LeCLERQ, La Fédération (janvier-juillet 1790), Paris, Letouzey et Aîné, 1929 ; aucun des articles publiés dans le livre de Jean EHRARD et Paul VIALLANEIX, Les fêtes de la Révolution : colloque de Clermont-Ferrand (juin 1794), Paris, Société des études robespierristes, 1977, ne traite en détail la Fédération en province. On trouvera un résumé du mouvement de la fédération en province dans Michel Vovelle, La découverte de la politique : géopolitique de la Révolution française, Paris, La Découverte, 1993, p. 38-44 ; et Yoichi URIO, «Espace et Révolution : enquête, grande peur et fédérations », AHRF, 280, 1990, p.157-160.

(5) Marcel DAVID, Fraternité et Révolution française, Paris, Aubier, 1987, notamment p. 4359. ; Haim BURSTIN, «Le "protagoniste" comme facteur d'amplification de l'événement : le cas de la Révolution française », dans Philippe JOUTARD (dir.), L'Événement, Marseille, Laffitte, 1986, p. 65-76 et La Révolution à l'œuvre. Le faubourg Saint-Marcel, 1789-1794, Paris, Champ Vallon, 2005 ; Jules Michelet, Histoire de la Révolution française, Paris, Laffont, 1979, vol.1, p. 318-340.

(6) Gustave LE Bon, La Psychologie des foules, Paris, Alcan, 1895.

(7) Georges RudÉ, La foule dans la Révolution française, Paris, Maspéro, 1982 ; Charles TILly, The Contentious French, Harvard University Press, Cambridge, MA, 1986. Id., La France conteste: de 1600 à nos jours, trad.fr. Paris, Fayard, 1986.

(8) A.R. ZoberG, « Moments of Madness » Politics and Society 2, n 2 (March 1972), p. 183-207; Sidney TARROw, « Cycles of Collective Action : Between Moments of Madness and the 
qui possèdent une « conscience collective », les émotions forment une base inévitable de connaissance ${ }^{9}$. Plus généralement, comme l'ont montré un nombre grandissant de travaux ces dernières années, les émotions sont partout : comprendre n'importe quel événement nécessite d'en assimiler les fondements émotionnels, parfois très banals, parfois plus complexes, sur lesquels un mouvement se construit ${ }^{10}$. L'émotion et la rationalité ne sont plus alors considérées comme des facteurs strictement contradictoires, mais comme des éléments qui se renforcent mutuellement ${ }^{11}$.

Dans cet article, nous allons examiner le développement du mouvement des fédérations, depuis les fédérations locales de 1789 jusqu' aux célébrations nationales du 14 juillet 1790, point culminant de la mobilisation, en passant par la vague de fédérations régionales au cours de l'année 1790. Ce faisant, nous attacherons une attention particulière aux facteurs qui ont rendu ces fêtes tellement riches en émotions et par là même tellement populaires. Pourquoi, malgré les nombreux problèmes que devaient résoudre les révolutionnaires dans les premiers mois de la Révolution, un grand nombre d'entre eux a-t-il consacré son énergie à organiser des rassemblements gigantesques attirant souvent jusqu'à des centaines de milliers de personnes ? Comment de telles manifestations émotionnelles ont-elles conduit les Français à participer à la Révolution, et ce faisant à s'identifier encore davantage à la nation française ?

\section{Les premières fédérations}

Les premiers temps de la Révolution ont été marqués par une atmosphère de grande incertitude et de forte intensité émotionnelle. Ils ont suscité à la fois l'espoir grandissant de changements, mais également la crainte des troubles et celle de la contre-révolution. Le mouvement des fédérations est le résultat direct du mécontentement lié aux troubles

Repertoire of Contention », Social Science History 17, n 2 (Summer 1993), p. 281-307. Sur le pouvoir mobilisateur de la peur, voir notamment Corey RoBIn, Fear: The History of a Political Idea, Oxford University Press, Oxford, 2004.

(9) Jon ElSTER, Sour Grapes: Studies in the Subversion of Rationality, Cambridge, Cambridge University Press, 1985 ; id., Alchemies of the Mind: Rationality and the Emotions, Cambridge, Cambridge University Press, 1999.

(10) Jeff GoODWIN, James M. JASPER and Francesca POLleTA (dir.), Passionate Politics: Emotions and Social Movements, Chicago, University of Chicago Press, 2001; Ronald AMINZADE and Doug MCADAM (dir.), « Special Issue on Emotions and Contentious Politics », Mobilization 7, $\mathrm{n}^{\circ} 2$ (Summer 2002); Helena FlAM and Debra KING (dir.), Emotions and Social Movements, New York: Routledge, 2005.

(11) Ronald AminZadE and Doug MCAdAM, «Emotions and Contentious Politics » Mobilization, p. 107-110. 
qui touchent tout le pays au cours de l'été 1789. Certes, de nombreux soulèvements remontent aux crises prérévolutionnaires de 1788 mais la première année de la Révolution connaît son lot de révoltes antinobiliaires locales, de peurs récurrentes et de mouvements liés aux subsistances, sans oublier les paniques de la Grande Peur ${ }^{12}$. Avec la propagation de la révolution municipale de juillet et août 1789, les autorités d'Ancien Régime sont renversées et c'est aux citoyens actifs que revient la responsabilité d'organiser le maintien de l'ordre et de mettre en place les nouvelles réglementations administratives. Les milices locales - bientôt institutionnalisées sur l'ensemble du territoire français sous l'appellation de gardes nationales - s'imposent pour rétablir le contrôle sur des populations qui refusent à la fois de continuer à reconnaître plus longtemps les autorités d'Ancien Régime, tout en se montrant sceptiques à l'égard des milices bourgeoises ${ }^{13}$. Dans ce contexte, les fédérations se sont rapidement révélées utiles pour maintenir une certaine cohésion dans le cadre instable des premiers temps de la Révolution.

Le 2 août 1789, dans la vallée de la Loire, la petite ville de Luynes célèbre au cours d'un rassemblement solennel, l'union des nouvelles autorités municipales à la garde nationale locale ${ }^{14}$. On le sait, les rassemblements de citoyens en armes avaient commencé au cours des mois précédents mais, pour la première fois, ce type de manifestation est présenté comme une confédération patriotique. Pour le nouveau comité municipal de la ville (composé de ses citoyens actifs) qui a concocté la cérémonie, il s'agit d'aider à consacrer le nouvel ordre dans des circonstances décrites en termes de «pénurie, insurrection et inquiétude générale ». "Considérant qu'aucune forme d'administration intérieure ne correspondrait mieux à une ville que celle qu'elle constitue elle-même », le comité a jugé que

(12) John MARKofF, The Abolition of Feudalism : Peasants, Lords and Legislators in the French Revolution, State College, PA, Pennsylvania State University Press, 1996 ; Georges LEFEBVRE, La Grande Peur de 1789, Paris, Armand Colin, 1932.

(13) Parmi de nombreux ouvrages traitant des soulèvements révolutionnaires provinciaux en 1789, voir notamment, Peter Michael JoNES, The Peasantry in the French Revolution, Cambridge, UK, Cambridge University Press, 1988, Anatoli ADO, Paysans en Révolution: terre, pouvoir et jacquerie, 1789-1794, Paris, Société des études robespierristes, 1996, Alan FORREST, Paris, the Provinces and the French Revolution, New York, Oxford University Press, 2004.

(14) Yonchi URIU, «Espace et révolution : enquête, Grande Peur, et fédérations », AHRF, 1990, p. 150-66. D’autres régions expérimentent des idées semblables : ainsi les membres de la garde nationale de Lons-le-Saunier proposent de « former une ligue offensive et défensive contre tous les ennemis de la nation », bien qu'aucune cérémonie ni aucun pacte ne semblent avoir été organisés par la suite. Danièle PINGUÉ, « Gardes bourgeoises et milices nationales en Haute-Saône, 1789-1790, » dans Serge BIANCHI et Roger DUPUY (dir.), La Garde nationale entre nation et peuple en armes, mythes et réalités, 1789-1871, Rennes, Presses universitaires de Rennes, 2006, p. 168. 
pareille affirmation collective de la solidarité révolutionnaire conviendrait au nouvel ordre. La cérémonie comprend plusieurs prestations de serments à « la Patrie, la Loi et le Roi », aux «droits de l'homme » et à la «sécurité publique » auxquelles prend part l'ensemble des participants ${ }^{15}$. Confrontés à une scène politique menacée par l'anarchie, les organisateurs des premières fédérations ont avant tout cherché à établir un contrepoint en travaillant à l'unité locale (et bientôt régionale et nationale).

Plus important encore au regard des développements ultérieurs du mouvement, les fédérés de Luynes publient une brochure reprenant le contenu de leur cérémonie et donnent ainsi à l'événement une large publicité relayée par la presse révolutionnaire naissante et les réseaux de correspondance. Même si le nombre d'observateurs extérieurs présents à Luynes pour la cérémonie fut très certainement limité voire nul, la manifestation fut connue grâce à ces publications. Rapidement, les descriptions de la fête inspirent d'autres cérémonies destinées à affirmer l'unité des gardes nationaux et des administrateurs locaux. Celles-ci prennent elles-aussi le nom de confédérations. Des fédérations commencent ainsi à s'organiser, non dans les seules villes de la Loire, mais dans le pays tout entier. Trois semaines après celle de Luynes, des confédérations apparaissent à Largentière (Ardèche) et Saint-Sauveur (Haute-Saône), deux villes situées à plus de 500 kilomètres de distance ${ }^{16}$. Dans le deuxième cas au moins, les organisateurs avaient formé un comité de correspondance destiné à « manifester leurs vœux et adhésions partout où besoin sera $\gg^{17}$. Les cérémonies sont pensées pour être universellement applicables, transposables et le plus largement partagées.

La force de tels événements reposant surtout sur le nombre de participants et l'affirmation collective d'objectifs communs, leurs organisateurs ont rapidement cherché à en étendre la taille et le contenu. Après les fédérations (et autres prestations de serments) essentiellement locales organisées entre la fin de l'été et le début de l'automne 1789, le mouvement est entré dans une phase nouvelle, celle des fédérations régionales, que symbolise la fédération de 12600 gardes nationaux à Étoile, dans la vallée du Rhône, le

(15) Acte de confédération patriotique et de Constitution provisoire de l'Administration, \& de la Milice citoyenne de la Ville \& Cité de Luynes (S.1., 1789), p. 1-3.

(16) Yonchi URIU, art. cit., p. 157 ; Henri BAUMONT, « Les fédérations dans la Haute-Saône et la fédération des quatorze villes bailliagères de Franche-Comté », Révolution française, 88, t. 14, p. 888-9. La fédération de Largentière eut lieu le 23 août, celle de Saint-Sauveur le 27 août.

(17) Henri BAUMONT, art. cit., p. 889. 
29 novembre ${ }^{18}$. À la différence des cérémonies de Luynes qui témoignent d'une mentalité plutôt défensive, celles d'Étoile cherchent, elles, à projeter la puissance du mouvement en avant, comme l'illustre le serment pris ce jour par les participants : « Nous, citoyens français des deux rives du Rhône de Valence à Pouzin, jurons d'être toujours unis [...] et de voler au secours de nos frères à Paris ou dans n'importe quelle ville de France où la liberté serait en danger $\gg^{19}$. Bien que la région concernée par la fête ne s'étende que sur vingt-six kilomètres, avec Valence comme seul centre de population important, les orateurs prennent soin de déclarer que l'événement s'applique à la nation tout entière, établissant ainsi un précédent pour des confédérations coopératives entre des régions plus larges.

La fédération d'Étoile donne lieu à la première publication de textes qui décrivent la force émotionnelle de ces cérémonies. Ces discours et témoignages sont rassemblés dans une brochure opportunément intitulée, Fédération proposée par la Garde nationale vivaroise de la Voûte, aux autres Gardes nationales françaises. Proclamant « nécessaire » l'unité contre les ennemis de la Révolution, le discours inaugural appelle à mettre en œuvre de nouvelles manières de « communiquer de proche en proche jusqu'aux confins de l'Empire, afin que d'un bout à l'autre de la France l'amitié fraternelle, la surveillance et l'appui mutuels » soient considérés comme les meilleurs moyens de sécuriser l'ordre nouveau ${ }^{20}$. Dans le texte qui suit, un commandant de la garde nationale déclare : "Aujourd'hui nos cœurs peuvent s'unir dans les mêmes affections, comme les eaux qui nous abreuvent s'unissent dans le même fleuve [...] Nous obéirons aux lois immuables de l'humanité, en faisant de chaque Corps national, le centre d'une alliance circulaire avec ses voisins $»^{21}$. En somme, ce commandant estime qu'en renforçant la réceptivité émotionnelle de la population par une reconnaissance réciproque et en éliminant les barrières qui divisent les partisans du projet révolutionnaire, il est possible de constituer une grande force collective.

Il est difficile de dire avec certitude pourquoi les rassemblements de soutien aux gardes nationaux se sont transformés en proclamations

(18) Gustave VALLIER, Essai sur les fédérations martiales en Dauphiné pendant les trois premières années de la Révolution française, Marseille, Olive, 1869, p.11.

(19) George LE GoRGEU, « Fragments entièrement inédits des AM de la ville de Vire. Fête de la Fédération du 14 juillet 1790, à Vire », A. Guérin, 1881.

(20) Fédération proposée par la Garde nationale vivaroise de la Voûte, aux autres Gardes nationales français (S.1, 1789), p. 4.

(21) « Discours de M. Ducluseau de Chabreuil, Chevalier de St. Louis, Colonel du Régiment des Gardes Nationales de la Ville de la Voute en Vivarais, prononcé aux Troupes réunies », dans Fédération proposée, p. 6-7. 
enthousiastes en faveur de l'égalité et de la fraternité révolutionnaires. Le sentimentalisme s'est certes développé dans la littérature au cours des décennies qui ont précédé la Révolution, mais ses manifestations françaises se sont essentiellement concentrées sur les actions individuelles ou interpersonnelles ${ }^{22}$. Et quand bien même l'amour du peuple a pu être proclamé par certains auteurs, il s'agissait d'un peuple encore abstrait que personne n'avait cherché à mobiliser directement. Même si le terme problématique de sentiment ou tout autre mot équivalent est souvent employé dans les récits de fêtes fédératives, il n'invoque pas explicitement des exemples littéraires ${ }^{23}$. Le mouvement des fédérations apparaît ainsi principalement comme une réaction aux événements présents : en réponse aux troubles qui affectent la plupart des provinces françaises en 1789, les révolutionnaires - qui cherchent à constituer un bloc solide face aux soulèvements paysans et aux contre-révolutionnaires en développant l'unité régionale et nationale - valorisent la force émotionnelle des grands rassemblements.

En repoussant les limites de leur nouveau moyen d'expression, les organisateurs locaux contribuent à rendre les fédérations de plus en plus grandes et de plus en plus ouvertes. Le 13 décembre, deux semaines après Étoile, la ville de Montélimar, située plus au sud dans la vallée du Rhône, accueille la première confédération interrégionale. Le nombre de participants est estimé à 6000 - soit moins de la moitié des participants à la fédération d'Étoile, mais cette fois la présence de délégués venus des régions voisines du Dauphiné, de Provence et de Languedoc, est très importante ${ }^{24}$. Pour la première fois, les fédérations suscitent l'intérêt de la presse nationale. Le Moniteur universel dans lequel sont retranscrits les débats de l'Assemblée nationale, évoque l'événement comme « une révolution aussi surprenante que la nôtre », dans laquelle « le peuple est armé mais les armes apportent la paix ». Qui plus est, le journal imagine quel rôle utile pourraient jouer les fédérations dans le projet révolutionnaire : «Le raisonnement ne console point, ne calme point, ne ramène point ; ce

(22) Sur le sentimentalisme français, voir David DENBY, Sentimental Narrative and the Social Order in France 1760-1820, Cambridge, UK, Cambridge University Press, 1994 ; Anne VINCENTBuffault, The History of Tears: Sensibility and Sentimentality in France, New York, St. Martin's, 1991 ; Pierre TRAHARD, La sensibilité révolutionnaire, Paris, Bolvin, 1936. Des concepts similaires ont été mis en œuvre plus tôt au cours du XVIII ${ }^{\mathrm{e}}$ siècle dans d'autres pays : voir Sarah Knott, Sensibility and the American Revolution, Chapel Hill, NC, University of North Carolina Press, 2009.

(23) Knott considère «l'ensemble des travaux dont la sensibilité fait partie » comme « notoirement vague », Ibidem., p. 50.

(24) Gustave VALLIER, op. cit. 
sont des faits qui touchent et persuadent $\gg^{25}$. En d'autres termes, pour être victorieuse, la Révolution doit être vécue.

Le grand rassemblement suivant se déroule à Pontivy, à plus de neuf cents kilomètres du Rhône. Cette fois il ne s'agit plus de faire bloc autour de la garde nationale locale mais plutôt de rassembler les « jeunes gens » des régions bretonne et angevine. Après avoir joué un rôle important dans les soulèvements de 1788 et 1789, notamment autour du Parlement de Rennes, les jeunes bretons cherchent à former une « coalition indissoluble », avec la conviction qu'un grand rassemblement positif « [frapperait] de terreur les ennemis de la régénération présente » tout en offrant de « nouvelles preuves d'un zèle » pour la Révolution. Leur rassemblement a d'ailleurs tout d'un effort constructif, avec la volonté de « consacrer par un acte authentique [...] l'heureuse Constitution qui vient de rétablir en France l'égalité sociale et la liberté », et démontre leur désir et leur intention de faire de la Révolution une réalité durable ${ }^{26}$. La participation au rassemblement n'excède pas 4000 personnes, mais ces jeunes qui ont de l'ambition déclarent en représenter $250000^{27}$.

Le groupe à l'origine de la fédération considérait son action comme un acte politique et non simplement symbolique. Grâce à la correspondance qu'il entretenait avec d'autres fédérés, notamment dans la vallée du Rhône, ce groupe pouvait s'exprimer avec assurance. Au cours du rassemblement, les jeunes annoncèrent que leurs compatriotes allaient converger sur Valence pour la troisième plus grosse fédération de la région. La nouvelle du rassemblement fut rapidement relayée par les multiples feuilles et journaux provinciaux. Les articles rédigés à cette occasion évoquent également des projets pour une éventuelle fédération nationale à Paris réunissant douze à quinze mille délégués de chaque fédération régionale - bien qu'à Paris personne n'eût encore donné son accord à un tel projet ${ }^{28}$. De toute évidence, au commencement de la diffusion apparemment spontanée du mouvement, la fédération n’était dominée par aucune élite spécifique (ni aucun autre groupe de partisans).

(25) Moniteur universel, 24 décembre 1789.

(26) Pacte d'union passé entre les jeunes citoyens de Bretagne et d'Anjou, réunis à Pontivy le 19 janvier 1790 (S.1., 1790) ; Procès-verbal des séances tenues par les Jeunes-citoyens de Bretagne et d'Anjou, extraordinairement assemblés en la Ville de Pontivy le 25 janvier 1790, s.1, Baudouin, s.d., 1. Voir aussi Nicolas DEPLANCHE, «From Young People to Young Citizens : The Emergence of a Revolutionary Youth in France, 1788-1790», Journal of Social History, 45, n¹ (2011), p. 225-237.

(27) Observateur provincial, vol. 2, n³.

(28) Le Nouvelliste national ou journal de Toulouse, politique, libre et impartial, 2 février 1790 ; Observateur provincial, vol. 2, n³. 
Avec près de 9000 gardes nationaux, la fédération régionale de Valence du 31 janvier 1790 se situe en nombre entre celle de l'Étoile et celle de Montélimar. Mais pour la première fois, en plus des participants directs, les récits mentionnent la présence d'une foule de spectateurs estimée à 30000 personnes. De fait, dans le but annoncé d'attirer le public le plus large possible, les organisateurs avaient organisé la fête sur un champ situé juste en dehors de la ville et utilisé habituellement pour les parades militaires. Outre l'objectif de faire naître la solidarité entre les soldats, les cérémonies visent désormais à créer « un spectacle très imposant » tant pour les participants que pour les spectateurs ${ }^{29}$. L'esthétique de la fête suscita des commentaires enthousiastes : des contemporains évoquèrent un spectacle qui transcende la beauté de la revue militaire et fait naître des émotions sublimes ${ }^{30}$. Pour le correspondant du Courrier de Paris qui avait observé la scène depuis les murs et les toits de la ville, « tout cet ensemble présentait à l'œil le tableau le plus sublime et le plus imposant qu'on puisse imaginer $»^{31}$. Désormais, la pièce ne s'adresse plus aux seuls participants, mais à la communauté tout entière.

Le rôle continu joué par les pays rhodaniens, au sens large, à la pointe du mouvement des fédérations, s'explique probablement à la fois par les soulèvements réels et les troubles potentiels auxquels la région était confrontée. Beaucoup craignaient que le royaume de Piémont-Savoie - qui hébergeait alors le Comte d'Artois et d'autres aristocrates contrerévolutionnaires - ne lance une invasion sur plusieurs fronts à travers les Alpes $^{32}$. Des heurts dans les campagnes avaient conduit à la proclamation de la loi martiale sur le Vivarais en décembre $1789^{33}$. Des confédérations plus petites faisant la promotion de la défense mutuelle s'étaient brusquement multipliées à travers la région dans les semaines suivantes, avec des rassemblements dans les villes de La Voûte, Dieu-le-Fit, Saint-Paul-lesChâteaux, Nyons, Romans, Privas et Puy-en-Velay, réunissant de 600 à 5 000 participants $^{34}$. Les zones déjà fédérées envoyèrent des représentants à chacun de ces rassemblements, rattachant ainsi de nouvelles fédérations au mouvement, et réciproquement chaque rassemblement faisait part de son

(29) Observateur provincial, vol. 2, nº ; Courrier de Paris, 12 février 1790.

(30) À bien des égards les catégories utilisées par les témoins reflètent celles développées par Edmund BURKE, Recherche philosophique sur l'origine de nos idées du sublime et du beau, 1769.

(31) Courrier de Paris, 12 février 1790.

(32) Gazette de Sainte-Lucie, 23 février 1790.

(33) Courrier français, 1er janvier 1790.

(34) Gustave VAlliER, op. cit., p.11-18; Patriote français, 12 et 18 février 1790. 
soutien et de son adhésion aux autres groupes fédérés ${ }^{35}$. À la mi-février, pas moins de quatorze fédérations s'étaient déroulées dans la vallée. La peur et les menaces grandissantes avaient poussé les révolutionnaires locaux à faire montre d'une plus grande fraternité et solidarité.

L'aspiration à de nouvelles fédérations, plus larges, se manifeste aussi dans l'ouest de la France. Le 15 février, soit quatre semaines après la fédération des jeunes gens, les gardes nationaux et les administrateurs municipaux de Bretagne et d'Anjou convergent vers la même ville pour un rassemblement destiné à « accélérer l'exécution des décrets de l'Assemblée nationale », et sont rejoints « en concert » par d'autres représentants pour discuter de sujets de préoccupations communs et pour signer un pacte fédératif ${ }^{36}$. Dans son élan, la municipalité d'Angers envoie une circulaire aux villes environnantes dans laquelle il est expliqué que les fédérations ne sont réussies qu'à la condition qu'elles soient actives : ceux qui y sont associés doivent continuer à «se transmettre leurs découvertes et se prêter des secours » pour soutenir de nouveaux rassemblements. Les partisans des fédérations y sont appelés à étendre le mouvement « répandant dans toutes les provinces ces sentiments d'union et de fraternité ${ }^{37}$. Et c'est ainsi que progressivement le statut des fêtes fédératives s'est transformé, substituant à l'objectif immédiat de solidarité et d'assistance mutuelle au niveau local, le projet d'un mouvement aux ambitions plus larges.

Toutefois, les fédérations ne constituent qu'un aspect des nombreuses fêtes et des cérémonies qui se déroulent à travers tout le pays en écho aux progrès de la Révolution. Tandis que la plupart des provinces n'ont pas encore formellement organisé de fédération de grande envergure, l'acceptation de la Constitution par le roi donne lieu à des prestations de serment dans tout le pays. Cette fois, comme le rapporte un journaliste témoin de la scène à Paris, ce ne sont plus seulement les gardes nationaux qui prêtent serment au nouvel ordre révolutionnaire, mais tous types de gens : «Les femmes, les enfants, les ouvriers, les domestiques, et les hommes de tous les rangs, de tous états, s'empressent de témoigner leur adhésion ». Alors que les serments parisiens sont prêtés au niveau du district, la cérémonie rouennaise concerne, elle, toute la ville : « un Peuple immense accourut à la Cathédrale, encore pénétré des douces émotions que lui avait procuré le matin le Discours du Roi et un Te Deum suivit la

(35) Fédération de Dieu-le-Fit en Dauphiné, s.1., s.d., p.1, p.10.

(36) AD Morbihan, L 1629 32, registre municipal de Fougères, 11 février 1790 ; AM Vannes, 2 I 136, circulaire (sans nom) de la municipalité d'Angers, 5 mars 1790.

(37) AM Vannes, Ibidem. 
cérémonie civique ». Les récits en provenance de Normandie insistent sur les sentiments fraternels qui, irrésistiblement, semblent s'imposer à tous les participants ${ }^{38}$. Quand bien même la population défère en partie aux autorités révolutionnaires, les festivités n'en sont pas moins marquées par une participation et une célébration largement collectives.

Le format et l'esprit des premières fédérations inspirent également des rassemblements hebdomadaires plus ordinaires. Au printemps 1790, l'Observateur provincial décrit ainsi l'exemple de la municipalité de Coulommiers-en-Brie où chaque dimanche, le maire, les officiers municipaux et la population se rassemblent dans l'église locale pour une lecture des derniers décrets de l'Assemblée nationale, s'instruisent également « des derniers complots des ennemis du bien public », et «finissent avec une exhortation à la concorde, à la paix et à l'attachement à la Constitution $»^{39}$. Comme pour les fédérations, il s'agit de favoriser à la fois la vigilance et l'attachement fraternel pour assurer efficacement et la survie et le développement de la Révolution.

Les fédérations se sont ainsi développées comme une réponse aux premières tensions de la Révolution et à l'espoir extatique que beaucoup plaçaient dans la nouvelle ère. Les peurs ont bel et bien participé à l'intensification des émotions des participants, conduisant les révolutionnaires à élaborer des proclamations de plus en plus élaborées de fraternité, de solidarité et d'amour. Bien que de nombreuses cérémonies aient été organisées en grande partie en réaction à des menaces extérieures, le succès du format des fédérations a dépassé les considérations strictement pragmatiques à mesure que la force émotionnelle des cérémonies s'est diffusée dans l'opinion. Une nouvelle conception de la nation française, forgée dans l'affirmation et la participation collectives de ses habitants, s'est développée. Encouragé par la publication de récits de fêtes fédératives toujours plus nombreux, un nombre plus grand de territoires a commencé à planifier ses propres célébrations.

\section{L'amplification du mouvement fédératif}

L'intensité, le nombre et la taille des rassemblements fédératifs ont continué à augmenter au cours de l'hiver et du printemps $1790 . \mathrm{La}$

(38) Observateur provincial, vol. $2, \mathrm{n}^{\circ} 6:$ : La joie brilla dans tous les yeux, chacun se félicitait, chaque regard exprimait un sentiment d'amour » [...] on a remarqué bien peu de Citoyens qui ne se soient empressés de partager ce témoignage de l'allégresse publique ». Pour Paris, voir Annales patriotiques et littéraires, 6 février 1790.

(39) Observateur provincial, vol. $3, \mathrm{n}^{\circ} 11$. 
presse a beaucoup contribué à attiser l'intérêt des populations pour ces fêtes : «Les actes fédératifs se multiplient de toutes parts », écrit en février le Journal patriotique de Bourgogne, « cette masse de force, cette unité d'opinion, doivent faire trembler les ennemis du bien et renverser leurs perfides projets $\gg^{40}$. Les manifestations de plus en plus importantes au cours desquelles le peuple fait la démonstration de son unité et de son attachement émotionnel s'imposent comme un moyen efficace pour amplifier la Révolution.

Les fédérations provinciales de mars 1790 organisées à Épinal (Vosges) et Agen (Lot-et-Garonne), deux chefs-lieux de départements distants l'un de l'autre d'environ 900 kilomètres, sont l'occasion d'évoquer la réalisation prochaine d'une fédération nationale : "Que l'époque », déclare le commandant des gardes nationaux d'Agen, « soit consacrée par une fédération solennelle entre toutes les milices patriotiques de chaque département, et qu'une chaîne fraternelle s'étendant d'un bout de la France à l'autre », finisse par former « une seule masse de forces et de volontés » pour soutenir la Révolution. Dans le même esprit, un témoin décrit la fédération d'Épinal comme la réalisation « de chaînes d'amour patriotique » entre différentes régions. Pour le commandant agenais, «L'association garantit le maintien de la tranquillité publique » par la force de la fraternitét ${ }^{4}$. Chaque fédération ne forme explicitement qu'une partie d'un processus à l'œuvre : chaque fête fédérative est l'occasion de rédiger des adresses aux autres provinces, invitant les participants à s'associer aux futures confédérations ${ }^{42}$. Ce faisant, un réseau fédératif se développe, incorporant des territoires de plus en plus larges au sein d'un projet national.

Pour ses partisans, le mouvement fédératif est un mouvement social de type nouveau. "Les grands mouvements politiques », écrivent les gardes nationaux d'Aix-en-Provence en avril 1790, sont le fait de « vrais citoyens [qui] forment une coalition pour la cause commune ». Dorénavant, à la différence de «l'apathie déshonorante » passée, l'action collective est à même de forger une nouvelle réalité. L'absence de précédent leur apparaît donc comme une force supplémentaire : en faisant la démonstration publique du «patriotisme et du zèle », ils espèrent « étonner les ennemis de la chose publique », désamorçant ainsi la nécessité d'un conflit direct ${ }^{43}$.

(40) Journal patriotique de Bourgogne, $\mathrm{n}^{\circ} 6$, février 1790.

(41) Fédération patriotique et militaire proposée aux milices nationales du Département du Lot et Garonne; par le régiment patriotique d'Agen, Agen, Noubel, s.d., p. 10-12.

(42) Ibidem, p.1.

(43) AM Aix-en-Provence, LL 203, Motion faite dans la Compagnie N. 26 de la Garde Nationale d'Aix, le 25 avril 1790, \& Délibération du même jour, s. 1., 1790, p.1-4. 
L'organisation de ces cérémonies est loin d'être facile. Henri Perchellet, garde national de la commune de Pithiviers, a raconté dans son journal les négociations qui ont précédé la fédération dans sa région. Ce sont les gardes nationaux d'Orléans qui ont pris l'initiative, en pointant la « solidarité » que ne manquerait pas de créer une telle fête et sa nécessité pour la sécurité de l'Orléanais. La question de l'hébergement des participants a donné lieu à controverse à cause du nombre très limité d'habitants volontaires pour accueillir les visiteurs, contraignant la municipalité d'Orléans à décréter que tous les « bourgeois et commerçants » de la ville hébergeraient au moins deux participants, à la condition cependant qu'ils puissent choisir lesquels. Parmi les gardes nationaux de Pithiviers, certains se portèrent volontaires pour participer, tandis que d'autres furent expressément mandatés par les autorités municipales. Malgré la météo exécrable le jour même de la fête, et des pressions diverses, Perchellet garde de la fédération une impression très favorable : "Les sentiments fraternels et patriotiques nous animent tous » écrit-il dès le lendemain ${ }^{44}$. Son témoignage souligne combien la force émotionnelle des fédérations a assuré leur succès.

Les partisans des fédérations ont poursuivi leurs efforts en faveur de cérémonies toujours plus importantes pour un public toujours plus large. À la fin du mois d'avril, l'Assemblée nationale reçoit des pétitions appelant à la mise en œuvre d'une fête fédérative nationale. La province cherche désormais à placer son mouvement au centre de l'État. Au terme de la fédération de Metz du 4 mai 1790, les fédérés s'adressent à l'Assemblée et insistent sur la puissance des fédérations : «il n'est pas un de nous qui ne soit pénétré de la force et de l'étendue de ces engagements; il n'en est pas un dans le cœur duquel ils ne soient écrits en caractères de feu $»^{45}$. L'intention pédagogique et la dimension émotionnelle ont conduit les partisans à promouvoir l'idée d'une fête d'ampleur nationale. "Que toutes les fédérations partielles », écrit la Garde nationale de Rennes, « soient aussitôt confondues dans une fédération générale ». Alors seulement, soutiennent-ils, « les ennemis extérieurs respecteront le repos d'un peuple belliqueux $»^{4}$. En présentant leurs rassemblements comme des mobilisations à la fois nécessaires et inévitables, les partisans des

(44) Henri PERCHELlet, Journal d'un bourgeois de Pithiviers pendant la Révolution française, Pithiviers, Imprimerie moderne, 1933, p. 405, p. 429, p. 449 et p. 452.

(45) Fédération de la ville de Metz, du 4 mai 1790, Metz, Lamort, s.d., p. 33.

(46) Lettre des commissaires de la garde nationale de Rennes, relative au projet d'une confédération générale des gardes nationales du royaume, datée du 29 avril 1790, S.1., s.d, p. 6. 
fédérations ne laissent pas d'autre choix à l'Assemblée nationale que celui de leur apporter son soutien.

Le nombre de gardes nationaux présents dans une fête fédérative atteint un maximum de 9000 soldats lors des premières fédérations du printemps. Le nombre de spectateurs continue, lui, à augmenter. Ainsi, la fédération de Nancy du 19 avril aurait attiré « un nombre infini de tous rangs, d'âge et de sexe $\gg^{47}$. L'ampleur sans précédent des manifestations semble avoir incité les organisateurs à recruter de plus en plus loin : « Dans quelle histoire, interroge le Journal patriotique de Bourgogne, at-on vu des exemples de conjurations formées par cent et cent cinquante mille personnes ? $\gg^{48}$. Aussi, pour augmenter la participation populaire, les organisateurs parrainent-ils des manifestations en amont et en aval de la fête principale. Perchellet décrit comment à l'issue de la fédération d'Orléans, les groupes participants se dirigèrent vers la place principale de la ville pour danser ${ }^{49}$. La fédération de Troyes, le 9 mai, a lieu au cœur d'une séquence de quatre jours de festivités populaires, qui coûtent la coquette somme de trente mille livres ${ }^{50}$. Outre le fait d'ouvrir le spectacle à des populations plus larges, les organisateurs prennent maintenant des mesures considérables et onéreuses pour toucher des foules plus importantes que jamais.

Tandis que le mouvement des fédérations continue sa progression, les récits se mettent à théoriser sur les effets possibles de ces rassemblements sur les populations participantes, proclamant notamment le pouvoir des sentiments. Par « les signes extérieurs de sa joie », écrivent les officiers municipaux de la ville de Challans à propos de leur fédération du 13 mai, « elle annonce d'une manière non équivoque, combien la cause qui rassembloit des hommes dignes de la liberté étoit chère à leurs cœurs ». En préjugeant d'une élimination totale de la distinction entre émotions extérieures et sentiments intérieurs, les leaders des fédérations révèlent à quel point ils sont convaincus par leur propre rhétorique de régénération révolutionnaire. À leurs yeux, l'ivresse provoquée par ces rassemblements doit permettre à des formes d'attachement fraternel plus radicales et plus profondes de s'épanouir ${ }^{51}$. À quatre jours de leur fédération, les organisateurs rennais ne manquent pas de souligner la rupture instaurée par la fédération avec les fêtes d'Ancien Régime : « Donnons à l'univers étonné le

(47) Affiche des évêchés de Lorraine, 29 avril 1790.

(48) Journal patriotique de Bourgogne, 6 avril 1790.

(49) Henri PERCHELLET, op. cit., p. 452.

(50) Chronique auxerroise, journal en manuscrit, AD Yonne, L 1402 93, 14 mai 1790.

(51) Procès-verbal de la Confédération de Challans, S.1., s.d., 6, 7, 13. 
spectacle imposant d'un Peuple de Frères libres et égaux » volontairement massés ensemble dans des proportions inédites ${ }^{52}$.

Les fêtes fédératives ne sont pas seulement euphoriques, elles sont également ouvertement religieuses. Les rassemblements sont présidés, au moins en partie, par un prêtre, et beaucoup comprennent une messe. D'ailleurs, la plupart des acteurs qui témoignent ne voient pas de contradiction entre les cérémonies catholiques et les fêtes révolutionnaires, à l'image de cet orateur auxonnais qui qualifie la fédération de Dijon de «magnifique harmonie de la religion et de la politique $»^{53}$. Ainsi, n'y a-t-il rien d'étonnant à ce que la fédération d'Auxerre du 2 juin ait débuté dans la cathédrale de la ville, après quoi les participants ont porté l'autel jusqu'au champ de parade à l'extérieur de la ville pour la deuxième partie de la fête. Les serments à la Révolution sont explicitement des « serments sacrés $»^{54}$. Pour certains participants, la Révolution ouvrait même la possibilité d'un christianisme régénéré : un retour à ce que le prêtre bordelais, qui officia lors de la fédération de Bordeaux avec Toulouse le 17 juin, décrit comme « l'union et la fraternité » enseignées par le Christ ${ }^{55}$.

Pour gagner tous ceux qui hésitent encore à apporter leur soutien à la Révolution, les fédérations multiplient les arguments et s'attachent à construire un consensus au sein des révolutionnaires divisés. "Quelle a été la source des désordres [de l'année dernière] ? L'ignorance et le défaut d'instruction », déclare un officier de la Garde nationale d'Auxonne à la fédération de Dijon le 18 mai. En plus de vouloir faire trembler la ContreRévolution, les fédérations entendent « prononcer au peuple l'éducation nationale qui lui manque ${ }^{56}$. L'émotion pourrait-elle permettre au peuple d'accéder à un niveau de compréhension supérieur ? Les fêtes fédératives sont aussi organisées à proximité des frontières, le long du Rhin ou dans les Alpes afin de séduire autant qu'intimider les populations étrangères ${ }^{57}$. D'un point de vue pédagogique, les fédérations fournissent un modèle de ce que peut signifier «être révolutionnaire». Un tel modèle a aussi

(52) Procès-verbal de la Fédération faite à Rennes le 23 mai 1790, entre la Garnison \& la Garde nationale de la même ville, Rennes, Vatar et Brulé de Remeur, 1790, p. 2.

(53) Ibidem, p. 78.

(54) AD l'Yonne, L 140297.

(55) AM Bordeaux, D 86 27. Sur les liens plus larges entre religion et fêtes révolutionnaires, voir Joseph CLARKE, Commemorating the Dead in Revolutionary France: Revolution and Remembrance, 1789-1799, Cambridge, UK, Cambridge University Press, 2007.

(56) Procès-verbal de la Confédération des gardes nationales des quatre départements formant ci-devant la Province de Bourgogne et pays adjacents, Faite sous les murs de Dijon, le 18 mai 1790, Dijon, 1790, p. 67-68.

(57) Annales patriotiques et littéraires, 1er mai 1790. 
une dimension normative, utile contre les non-conformistes : la diffusion rapide des idées démocratiques et le développement des sociétés de pensée grâce à la multiplication des réseaux de clubs inquiètent beaucoup ceux qui craignent que le fossé politique entre les révolutionnaires ne s'aggrave. Pour le rouennais Gosselin, les fédérations constituent un moyen efficace pour d'une part, faire oublier les désaccords chez les révolutionnaires et d'autre part, « resserrer les liens entre les bons citoyens et isoler ceux qui ne [veulent] pas concourir à l'œuvre commune $»^{58}$. En ce sens, si les fédérations portent les aspirations à l'unité du mouvement révolutionnaire, elles se construisent également contre les opposants à la Révolution à l'extérieur et contre les non-conformistes à l'intérieur.

En effet, le développement d'un réseau de clubs jacobins provinciaux intervient dans une séquence chronologique très proche de celle des fédérations, avec des justifications qui se recoupent. Les deux mouvements cherchent à créer des associations fortes pour l'éducation, le partage d'informations et enfin l'assistance mutuelle contre les menaces qui pèsent sur la Révolution. «Dans ce moment » de confédération, écrit en juin 1790 un membre du club de Pontoise aux jacobins de Cherbourg, les jacobins ont tenté de créer dans leur sillage "les nœuds d'une alliance aussi sainte et aussi utile pour la Patrie $»^{59}$. En mai, les jacobins de Béziers déclaraient l'affiliation de leur club à celui de Montpellier afin de faire partie de leur "pacte fédératif $»^{60}$. Tandis que les fédérations encouragent les municipalités et les gardes nationaux à correspondre, à échanger sur des sujets de préoccupations communs, les jacobins s'orientent eux vers la création d'un réseau qui échappe au contrôle politique du gouvernement. Bien que les premiers clubs n'aient été créés qu'en novembre 1789, on compte à la fin de l'année 1790 , plus de trois cents sociétés affiliées réparties sur tout le territoire ${ }^{61}$.

Le modèle fédératif continue de gagner du terrain. Le 30 mai, la fédération de Lyon est la première à manifester des aspirations nationales. Des délégations en provenance de tout le pays se joignent aux quelques cinquante à soixante mille fédérés locaux sous les yeux d'un nombre encore

(58) Édouard Hippolyte GossELIN, Journal des principaux épisodes de l'époque révolutionnaire, à Rouen et dans les environs de 1789 à 1795, Rouen, Cagniard, 1867, p. 43.

(59) AM Cherbourg 2 I 1271.

(60) AD Hérault, L 5532.

(61) Serge Bonin et Claude LANGLOIS (dir.), Atlas de la Révolution française : Sociétés politiques, Vol. 6, p. 34 ; Patrice HigONNET décrit l'esprit unanimiste des fédérations comme « protojacobin », Goodness Beyond Virtue : Jacobins during the French Revolution, Cambridge, MA, Harvard University Press, 1998, 25. 
plus grand de spectateurs ${ }^{62}$. À la différence des fédérations régionales précédentes, le maire de Lyon proclame celle-ci comme «la fédération universelle de toutes les parties de l'empire ». La participation à la cérémonie n'est pas limitée aux gardes nationaux, mais rassemble une assistance composée d'hommes et de femmes de tous âges. Là où auparavant seules les forces masculines avaient défilé, pour la première fois des unités féminines, « rappelant le courage de Jeanne d'Arc », rejoignent «sabre à la main » les rangs des participants. Enfin, alors que les fédérations d'adolescents s'étaient précédemment déroulées séparément, pour la première fois les jeunes défilent aux côtés des adultes ${ }^{63}$. Malgré la taille de la « vaste plaine » et les « superbes amphithéâtres » construits pour accueillir la foule, «l'affluence incroyable entraîna les spectateurs dans les immenses espaces » et les déborda ${ }^{64}$. «Bientôt, rapporte un observateur en se référant à la réaction extatique des foules rassemblées à Lyon, les François n'auront plus qu'une même âme $»^{65}$.

Le nombre de spectateurs excédant maintenant largement le nombre de participants, les récits des cérémonies se concentrent davantage sur la réaction populaire au spectacle de la fête qu'au spectacle en lui-même. «Le peuple ne doit pas être simple spectateur », peut-on lire dans le journal radical, Les Révolutions de Paris, dès le mois de février, « il doit être acteur ${ }^{66}$. Les nombreuses fédérations de juin dépassent celle de Lyon en taille. La fédération lilloise du 6 juin attire près de cent mille personnes. Dans son discours prononcé au terme du serment collectif, le maire de Lille décrit « un peuple d'amis », rassemblés par les transports « d'une joie réciproque » animant la foule qui se masse sous ses yeux ${ }^{67}$. «Jamais le soleil n'a brillé au-dessus d'un spectacle plus grandiose et imposant et plus digne de la majesté des nations » déclare L'Abeille patriote. Le journal décrit abondamment les effets produits par la fête sur l'assistance, «les yeux rendus humides par les larmes des sentiments », « les cœurs avec la joie la plus pure ${ }^{68}$. Une semaine plus tard, la fédération de Strasbourg attire 75000 personnes.

(62) Confédération de Lyon, le 30 mai 1790, Lyon, La Roche, 1790, p. 30, p. 34 ; Moniteur universel, 6 juin 1790.

(63) Ibidem, p. 2-3.

(64) Camp fédératif de Lyon; ou récit de tout ce qui s'est passé le jour de la fédération, \& les journées précédentes; Avec la description du Camp, l'ordre de la marche, l'adresse à l'Assemblée nationale, la formule du serment, où l'on a ajouté ce qui est arrivé de plus remarquable le lundi \& mardi suivants, Lyon, 1790, p.1.

(65) Relation du camp fédératif sous les murs de Lyon, le 30 mai 1790, Lyon, s.d., p.1.

(66) Les Révolutions de Paris, 13-20 février 1790.

(67) AD Nord, L 124449.

(68) L'Abeille patriote ou Feuille de tous les jours, 9 juin 1790. 
Le nombre grandissant de rassemblements, qui étonnamment ne s'accompagnent d'aucun débordement sérieux, démontre qu'il est possible d'organiser des actions collectives dans le calme. Le mouvement rassemblant les gardes nationaux et le peuple pour célébrer leur participation commune au projet révolutionnaire ne donne lieu à aucune protestation directe de la part des groupes conservateurs. Les journaux, pourtant enclins à commenter tout rassemblement en terme de conflit, s'étonnent à plusieurs reprises de l'absence même de contestation ouverte. Dans son récit de la fédération de Poitiers du 12 avril, le Patriote français souligne non sans une légère surprise combien « la joie qui y a régné n'a nui ni à l'ordre ni à la tranquillité ». Dans l'article qu'il consacre à la fête fédérative du 26 juin à Mont-de-Marsan, le Moniteur universel note lui aussi l'absence des « effervescences » qui généralement ne manquent pas de survenir lorsque de telles foules sont rassemblées ${ }^{69}$. Les rassemblements populaires, habituellement réprimés sous l'Ancien Régime, et toujours considérés avec suspicion au début de la Révolution, imposent progressivement leur légitimité.

Les limites des fêtes fédératives sont encore repoussées. Elles donnent désormais lieu à des événements encore plus ouverts et plus mobiles. Le défilé des troupes qui se rendent aux fédérations, notamment lorsqu'elles traversent des villes et des villages, devient un événement en soi. Un détachement rouennais en route pour la fédération de Chartres, au début du mois de juin, est ainsi fêté par des foules nombreuses dans les villes qu'il traverse, accueilli par «tous les bons patriotes, les officiers municipaux et complimenté par le maire en habit cérémonial ». L'enthousiasme des autorités et des populations est tel qu'il oblige les officiers du détachement à demander à la troupe de refuser les invitations à venir boire dans une «maison publique » locale. À Évreux, les gardes nationaux de la ville partirent en défilé à leur rencontre, sans armes, afin de « conserver leurs bras entièrement libres pour les y mieux recevoir ». La rencontre donna lieu à une fraternisation générale ${ }^{70}$. Enthousiasmés par l'émotion qui habite ces rassemblements, les municipalités, les gardes nationaux et l'ensemble des citoyens continuent à investir du temps et des ressources dans ce mouvement.

Au cours de la première moitié de l'année 1790, les fédérations deviennent donc un aspect majeur du projet révolutionnaire. Elles touchent

(69) Moniteur universel, 8 juillet 1790.

(70) François-Antoine DELAFOND, Itinéraire ou journal exact du détachement de la garde nationale de Rouen, qui s'est rendu à la Fédération de Chartres, rédigé et lu le 21 juin 1790, Rouen, Dumesnil, 1790, p. 5-7. 
pratiquement l'ensemble des provinces. Ce mouvement qui avait commencé comme un appel aux armes en soutien aux gardes nationales locales s'est mû en une impressionnante démonstration d'affirmation révolutionnaire qui intègre à différents titres pratiquement tous les groupes qui composent la société française. Tout en conservant leur caractère militaire, ces fêtes diffusent dans la société française les objectifs égalitaires et fraternels de la Révolution. Assez rapidement toutefois, les révolutionnaires commencent à s'y sentir à l'étroit, même lors des plus grands rassemblements régionaux dont l'ampleur est pourtant alors inédite : plus que jamais ils sont convaincus que la France toute entière a désormais besoin de se rassembler à l'occasion d'un acte collectif.

\section{La fête parisienne.}

Paris, qui s'est retrouvé tant de fois à l'avant-garde de la Révolution, met du temps à réagir au phénomène. À la fin de l'année 1789 et au début de l'année 1790, l'idée d'unir les provinces à l'occasion d'une grande cérémonie nationale ne suscite guère l'intérêt des hauts responsables de la municipalité, ni celui des députés de l'Assemblée nationale. Longtemps c'est la date encore lointaine et incertaine de la présentation de la nouvelle constitution qui a la faveur des chefs parisiens ${ }^{71}$. Vers la mi-janvier 1790, les autorités parisiennes commencent à planifier une prestation de serment pour le 14 juillet, mais uniquement destinée aux jeunes hommes de vingt et un ans commençant leur service au sein de la Garde nationale ${ }^{72}$. Ce n'est que sous la pression d'un actif lobbying de la province sur Paris, d'abord par la diffusion de brochures, de journaux et de lettres, puis à l'aide de délégations envoyées à la capitale, que l'idée d'une fête fédérative nationale finit par s'imposer. Après avoir fait ses preuves dans les provinces, le programme des fédérations va maintenant pouvoir s'imposer au sein du cœur politique de la nation.

La fédération bretonne-angevine est la première à avoir véritablement attiré l'attention de la capitale en envoyant à Paris une délégation qui se présenta le 20 mars devant l'Assemblée pour y lire l'accord passé entre les deux provinces. Celle-ci donna son approbation et le texte put être imprimé

(71) Timothy TACKETT, Par la volonté du peuple, op. cit. Le seul appel isolé pour l'organisation d'un tel rassemblement émane du district des Récollets le 17 novembre 1789, voir Sigismond LACROIX (dir.), Actes de la Commune de Paris pendant la Révolution, Paris, Cerf, 1897.

(72) Ibidem, Série I, vol.3, p. 417. 
et distribué dans les départements ${ }^{73}$. Le 29 mars, la délégation présenta une adresse bien plus radicale devant le club des jacobins de Paris, déclarant qu' « une fédération générale est le seul moyen d'affermir la Révolution ${ }^{74}$. Tandis que la position des députés sur la question n'était pas encore connue, l'intérêt de Paris pour le mouvement des fédérations grandissait. Pour rendre hommage aux fédérés de province, les soixante districts de la capitale organisèrent des rassemblements trois semaines plus tard et firent tirer un coup de canon à l'heure exacte où les participants à la fédération bretonne-angevine avaient prêté le serment ${ }^{75}$. Les Parisiens, qui jusqu'alors s'étaient méfiés du mouvement par crainte que ne se réalisent des alliances en dehors de l'État central, se mirent à envisager pour eux-mêmes ce genre de cérémonie.

$\mathrm{Au}$ cours du printemps, les districts parisiens commencent alors à militer en faveur d'une fédération nationale. Le 22 avril, le district de Saint-Honoré présente devant le conseil municipal de Paris une mesure qui fait explicitement référence à la fédération bretonne-angevine comme base pour une « confédération nationale », tout en suggérant que la participation soit limitée aux populations vivant à moins d'une journée de marche de Paris ${ }^{76}$. Malgré l'absence de décision de la municipalité, le district de Saint-Eustache envoie un courrier dans les villes de province dès le mois de mai pour mesurer l'intérêt d'une " fédération générale des provinces dans la capitale $\gg^{77}$. Quelques semaines plus tard, le district envoie un délégué participer à l'immense fédération de Lyon afin de créer des liens et probablement aussi de rapporter le maximum de détails sur l'organisation d'un événement d'une telle importance ${ }^{78}$. À Paris comme en province, ce ne sont pas les représentants du pouvoir central qui font campagne en faveur des fédérations, mais bien les organisations locales.

Les autorités parisiennes continuent à recevoir de nombreux récits faisant l'éloge des fédérations régionales. Par leur force de conviction, ces textes ressemblent parfois à des récits de conversion religieuse. Vers la mimai, en plus des écrits dont le nombre ne cesse d'augmenter, la fédération

(73) Lodoïs LATASTE et al (dir.), Les Archives parlementaires de 1787 à 1860 : un recueil complet des débats législatifs et politiques des Chambres françaises, Paris, Dupont, 1867, Séries 1, Vol. 12, p. 265. La première fédération de Pontivy s'était contentée de faire parvenir à l'Assemblée une communication écrite, cf Sigismond LACROIX, op. cit., Série I, vol. 5, p. 403.

(74) Alphonse AULARD, vol.1, p.58.

(75) Journal de la fédération générale, $\mathrm{n}^{\circ} 1$, s.d.

(76) Sigismond LACROIX, op.cit,. Série I, vol. 5, p. 114.

(77) AM Sens, 2 D 2 41. La municipalité de Sens fit rapidement part de son intérêt (2 D 2

$50)$.

(78) Confédération de Lyon, p. 36. 
d'Orléans décide d'envoyer une délégation à la municipalité de Paris. Ses orateurs se présentent devant les autorités et décrivent dans le détail les effets de leur fête fédérative sur les participants, l'énergie incroyable engendrée par l'événement, ou encore « la douce émotion qui remplit tous les cours $»^{79}$. Au cours du printemps 1790, il semble que Paris soit la seule grande ville du pays qui n'ait pas encore organisé de rassemblement de ce type. Étant donné la publicité donnée à ces événements, il devient difficile pour les responsables municipaux de refuser plus longtemps l'organisation d'une célébration nationale.

Dans le même temps, en province, le projet d'une fédération nationale qui se passerait du contrôle central de Paris fait de plus en plus d'adeptes. Le 11 mai, l'Assemblée nationale reçoit un courrier en provenance d'Arras annonçant deux confédérations jumelles, l'une à Arras, l'autre à Lille, organisées à l'intention des gardes nationaux des départements du Nord et du Pas-de-Calais. Les auteurs de ce courrier vont plus loin et appellent aussi à décider d'une date pour « une seule et même confédération générale » afin que tous les gardes nationaux de France puissent prêter le même serment à la même heure dans les chefs-lieux de leur département. Avec « quatre millions de mains [qui] se lèveraient vers le ciel », supposent-ils, «ce serment retentirait dans vingt-quatre millions de cœurs », touchant ainsi toute la population française ${ }^{80}$. La proposition est unanimement applaudie par les députés et immédiatement soumise aux comités de l'Assemblée... d'où elle n'est jamais réapparue ${ }^{81}$. La prétention des députés à représenter la souveraineté nationale aurait pu être ébranlée par une fédération nationale prenant place en dehors de leur contrôle.

L'ampleur de la mobilisation provinciale finit par contraindre l'Assemblée à organiser conjointement avec la municipalité de Paris une fédération nationale dans la capitale. «Tout est en train de changer autour de nous » déclare Jean-Sylvain Bailly, le maire de Paris, devant l'Assemblée le 5 juin, «quasiment toutes les régions de l'empire ont formé leur propre fédération ». Confronté à une telle unanimité, il s'adresse aux députés : « honorez votre suffrage » et terminez votre œuvre par un grand rassemblement national ${ }^{82}$. Malgré des objections venues des rangs conservateurs (le temps des gardes pourrait être mieux utilisé) la motion reçoit un large soutien. La taille du rassemblement serait inédite : l'Assemblée nationale

(79) Sigismond LACROIX, op.cit., Série I, vol. 5, p. 511.

(80) Archives parlementaires, Série I, vol.15, p. 488-489.

(81) Révolutions de Paris, 8 au 15 mai 1790.

(82) Le Postillon, 6 juin 1790. 
approuve le projet d'un stade long de 2700 pieds de chaque côté pouvant accueillir 252000 personnes - une capacité que n'atteignent aujourd'hui que de rares structures ${ }^{83}$. Les dimensions du lieu choisi pour la fête sont impressionnantes, ce qui n'empêche pas les partisans d'un rassemblement encore plus grand sur la Plaine des Sablons (à même de contenir jusqu'à huit à neuf cent mille personnes) d'exprimer leur mécontentement ${ }^{84}$. La fête en préparation s'inscrit délibérément sur une échelle sans précédent, même en comparaison avec les plus grandes fédérations régionales.

Les différents projets se retrouvent autour de l'idée d'une série de célébrations simultanées, à la fois à Paris et dans des villes périphériques à travers tout le pays, le 14 juillet 1790. Avec plus de 2,5 millions de gardes nationaux désignés pour participer, les organisateurs cherchent à attirer plusieurs fois ce nombre en spectateurs ${ }^{85}$. Les aspirations universalistes de la fête de la Fédération conduisent à appliquer la même formule partout : tous les Français sont invités à « s'engager personnellement ${ }^{86}$. Chaque municipalité doit organiser sa propre célébration. «Que le même jour, à la même heure, un cri général, un cri unanime se fasse entendre dans toutes les parties de la France », résume la proclamation officielle de l'Assemblée nationale ${ }^{87}$. En célébrant le premier anniversaire de la chute de la Bastille, les Français se retrouvent littéralement en position d'agir « de concert » comme un seul homme ${ }^{88}$.

Conformément au projet universaliste des premiers penseurs révolutionnaires, beaucoup des partisans du mouvement des fédérations croyaient que leur exemple ferait des émules au-delà des frontières du royaume. Dans un discours à l'Assemblée le 19 juin 1790, Anacharsis Cloots plaide ainsi pour que la fédération ne soit pas «seulement la fête des Français mais encore la fête du genre humain ». Le Baron de Menou qui préside la session déclare à son tour que les avancées de la Révolution « appartiennent

(83) Observations du sieur Blondel, architecte et dessinateur du Cabinet du roi, sur le projet de la confédération patriotique, du 14 juillet 1790, Paris, Imprimerie nationale, 1790, p. 6. La taille inédite des structures mises en chantier fut remarqué dès l'époque Lettres à $M . B^{* * *}$ à Genève, sur la fête de la Fédération nationale, célébrée à Paris le 14 juillet 1790, s.l., s.d., p. 2. De nos jours, seules les tribunes du circuit automobile d'Indianapolis (Indianapolis Motor Speedway) dans l'État de l'Indiana aux États-Unis sont susceptibles d'accueillir un plus grand nombre de spectateurs.

(84) Richard A. ETLIN, «Architecture and the Festival of Federation, Paris, 1790 » Architectural History18, 1975, p. 25-30.

(85) Un rapport de renseignement de l'ambassadeur de Prusse estime le nombre de gardes nationaux officiellement enregistrés à 2571 700. Geheime Staatarchiv (Berlin, Allemagne), Merseberg Rep. $92, \mathrm{n}^{\circ} 3$.

(86) AD Rhône, 1 L 369.

(87) Le Postillon, op.cit.

(88) Révolutions de Paris, 12 au 19 juin 1790. 
également à toutes les autres nations » et approuve la participation de contingents étrangers ${ }^{89}$. Ces révolutionnaires estiment que la régénération $\mathrm{du}$ monde est possible et qu'elle exige la participation de tous ${ }^{90}$. Afin de recruter des délégués britanniques pour la fête parisienne, les organisateurs envoient une invitation à la London Revolution Society ${ }^{91}$. Plus tard, un journal a alimenté à sa façon la croyance dans le pouvoir des fêtes en déclarant : «S'il s'étoit trouvé parmi nous un seul homme de chaque nation, au moment où la famille des Français a juré la liberté, et que cet homme, quel qu'il fût, retournât chez ses compatriotes, bientôt tous les tyrans auroient disparu ; nous avons donné à l'Univers le signal de la liberté $»^{92}$. Tout en participant à la construction de l'unité nationale, les révolutionnaires ont ainsi conçu avec les fédérations un modèle facilement transposable n'importe où ailleurs.

Ce ne fut pas facile d'être prêt dans les temps, notamment de terminer l'aménagement des lieux mêmes de la fête pour le 14 juillet. La préparation de l'événement avec la tension qui l'entourait demanda beaucoup d'efforts de la part des volontaires. La situation devint extrêmement préoccupante lorsque les ouvriers chargés de la construction menacèrent de faire grève, refusant de travailler jour et nuit jusqu'à la fin des travaux de construction du stade ${ }^{93}$. Les sections et les faubourgs de Paris, dans un grand élan de solidarité, mobilisèrent des dizaines de milliers de volontaires pour terminer l'aménagement de l'esplanade. L'identification émotionnelle avec le projet fédératif constituait un facteur de motivation de premier ordre : le nombre de volontaires qui se pressèrent sur le site dépassa même les besoins : «L'ardeur du public à travailler au Champ de Mars s'est répandue si généralement qu'il n'y avait peut-être pas un homme ni une femme dans Paris qui n'eut fortement l'envie d'y travailler » rapporte un parisien ${ }^{94}$. On estime le nombre de volontaires à plus de 125000 personnes $^{95}$. La ville prit alors le visage d' $\mathrm{d}^{\text {' }}$ un vaste atelier patriotique ${ }^{96}$. La démonstration

(89) Archives parlementaires, Série 1, vol. 16, p. 373.

(90) Pierre SERNA, « Toute révolution est guerre d'indépendance », dans Jean-Luc CHAPPEY, Bernard Gainot, Guillaume MAZEAU, Frédéric RÉGEnt et Pierre SERnA (dir.), Pour quoi faire la Révolution ?, Marseille, Agone, 2012, p.19-21.

(91) Richard PRICE, Correspondence, Durham, NC, Duke University Press, 1984, Vol. 3, p. 306.

(92) Confédération nationale, $\mathrm{n}^{\circ} 1$.

(93) Journal général de la cour et de la ville, 2 juillet 1790.

(94) Chantal PLANTIER-SANSON (éd.), Lettres d'un bourgeois de Paris à un ami de province, 1788-1793, Paris, 1993, p.144.

(95) Henri LECLERQ, La Fédération (Janvier-juillet 1790), op.cit., p. 321. Les estimations proposées à l'époque citent 120 000, 150 000, 200000 and 250-300 000.

(96) Observateur provincial, vol. 3, n²2 (12 juillet 1790). 
de bonne volonté de ces hommes et de ces femmes permet à la Fête de la fédération d'avoir des effets bien plus importants sur les populations que n'importe quelle directive.

Beaucoup s'inquiètent toutefois des désordres que le rassemblement serait susceptible de provoquer. «On dit qu'il y aura plus de quatre cent mille étrangers à Paris le 14 juillet prochain et il y a des gens qui s'en effrayent », écrit un député ${ }^{97}$. On estime à 44000 le nombre de délégués dépêchés par les municipalités françaises ${ }^{98}$. Les risques que des groupes cherchent à porter atteinte à la Révolution lors des cérémonies sont pris très au sérieux. Des milliers de gardes nationaux sont privés de fête et réquisitionnés pour sécuriser les alentours du Champ de Mars. Les autorités révolutionnaires sont conscientes du risque que représente la venue d'un nombre sans précédent d'étrangers dans la capitale et pourtant, à la vue de la force apparente et du nombre de leurs partisans, elles l'acceptent. Des manifestations ciblant de soi-disant contre-révolutionnaires troublèrent cependant les préparatifs de la fête. Des ouvriers se dirigeant vers le Champ de Mars entourèrent le carrosse de l'ambassadeur de Naples et le menacèrent aux cris d' « A bas la livrée! A bas les armoires! Les aristocrates à la lanterne ! ${ }^{99}$. Plus sérieusement, les parisiens tentèrent de lyncher un voleur capturé sur le site de la fête une semaine auparavant et furent arrêtés de justesse par la Garde nationale ${ }^{100}$. L'insécurité était toujours prégnante au sein de l'entreprise révolutionnaire, et les émotions intenses ressenties au cours de cette période pouvaient être dirigées contre des hommes perçus comme des opposants. Malgré les divisions qui caractérisent Paris, aucune fracture supplémentaire ne s'ouvre au cours des principaux moments de la fête de la Fédération.

La mobilisation dans la perspective de la fédération parisienne avait peu à peu gagné tout le pays avec l'élection dans chaque municipalité des gardes nationaux délégués pour la cérémonie nationale. L'Assemblée avait décidé de prendre en charge les frais de transports des délégués à hauteur de 300 livres, mais pour les représentants de nombreuses régions, le voyage

(97) Claude-Germain RoussElot, Correspondance de l'abbé Rousselot, constituant (17891795), Besançon, Université de Besançon, 1992, 76.

(98) Pierre DE VAISSIÈRE, Lettres d' «aristocrates». La Révolution racontée par des correspondances privées, 1789-1794, Paris, Perrin, 1906, p.172.

(99) Albert Mousset, Un témoin ignoré de la Révolution: le comte Fernan Nùnez, ambassadeur d'Espagne à Paris, 1787-1791, Paris, Champion, 1923, p.105.

(100) Journal général de la cour et de la ville, 8 juillet 1790. 
jusqu'à Paris signifiait aussi plusieurs semaines d'absence et par conséquent de travail perdu ${ }^{101}$. Néanmoins, l'événement attire une assistance importante, venue de toute la France. En chemin, comme à l'occasion des fédérations régionales, les participants sont accueillis chaleureusement par les municipalités situées sur les routes qui les emmènent à Paris et se voient offrir le gite et le couvert ${ }^{102}$. Partout la participation populaire massive et le volontarisme affichés par les autorités locales contribuent de façon essentielle au succès de l'événement.

L'après-midi du 13 juillet, malgré une forte pluie, près de quatorze mille gardes nationaux venus de province se massent place Louis XV pour un premier défilé à travers le jardin des Tuileries devant le roi et son palais ${ }^{103}$. Une délégation de vingt personnes issue des gardes nationaux de tout le territoire se joint à Lafayette pour présenter une adresse à l'Assemblée. Si la nation se trouve dans l'attente enthousiaste de la nouvelle constitution, explique ce dernier, la «volonté éclairée » qui la guide est déjà en train de créer un nouvel ordre. La fête, poursuit-il, sera complète si elle est l'occasion que soient proclamés de nouveaux idéaux révolutionnaires :

« Oui, Messieurs, nos mains s'élèveront ensemble à la même heure ; au même instant nos frères de toutes les parties du royaume profèreront le serment qui va les unir : avec quels transports nous déploierons à leurs yeux les bannières, gages de cette union et de l'inviolabilité de nos unions ! avec quels transports ils les recevront! Puisse la solennité de ce grand jour être le signal de la conciliation des parties, de l'oubli des ressentiments, de la paix et de la félicité publique !».

Le discours est applaudi chaleureusement ${ }^{104}$. Et en dépit de l'atmosphère de division qui avait entouré les premiers débats sur l'opportunité d'organiser une fédération nationale, tous les députés déclarent s'engager publiquement, avec un enthousiasme non dissimulé, dans les cérémonies.

Pour la célèbre fête du 14 juillet, le modèle des fédérations régionales est suivi à la lettre à l'exception de la prise de serment royale. Des dizaines de milliers de gardes nationaux défilent au centre d'un stade immense rempli de centaines de milliers de spectateurs. L'un d'entre eux a décrit son expérience dans les termes suivants : «Ce n'est pas dans un champ que l'on croit entrer, mais dans un autre monde », entouré d'une masse

(101) AD Drôme, L 2248.

(102) AM Chartres, C 111.

(103) Henri LECLERQ, La Fédération, op. cit., vol. 1, p. 335.

(104) Archives parlementaires, vol. 17, p. 77. 
sans précédent d'humanité ${ }^{105}$. Après une cérémonie religieuse, les gardes nationaux puis le roi prêtent serment. D'après un témoin, «le calme respectueux et ininterrompu » observé au cours des serments est rompu par un tonnerre d'applaudissements et d'embrassades. La scène donne lieu à « des sentiments puissants que les mots ne suffisent à décrire et dont l'imagination humaine, affectée par le spectacle grandiose qui s'offre à elle, ne peut former aucune conception adéquate ». L'émotion des uns et des autres s'exprime alors par des termes collectifs, et la joie ressentie est directement rapportée à l'ampleur de l'entente réalisée ${ }^{106}$. Un autre spectateur a raconté à ses proches avoir vu « cinq cent mille personnes animées d'un même esprit, d'une même intention, jurer au nom de tout un peuple $»^{107}$. Il se dit désormais convaincu que le mouvement des fédérations est « le plus sûr garant de [la] félicité et de la réalité de [la] régénération [à l'œuvre] »

Malgré la pluie, la bonne humeur règne. La longue procession à travers Paris depuis la place de la Bastille jusqu'au Champ de Mars est animée par les foules massées sur les balcons et les rebords de fenêtres, qui jettent du pain et distribuent de l'eau-de-vie aux participants. Un garde national du village de Sézanne (Marne) qui visitait Paris pour la première fois à cette occasion s'est déclaré profondément touché «par les amitiés que tous les Parisiens ne cessaient de témoigner par des applaudissements et des redoublements de vivent nos frères! vivent nos camarades! ${ }^{108}$. Un autre provincial décrit la fête comme un moment « non seulement imposant par sa beauté, mais encore consolant par les germes d'union et de fraternité que les députés ont apportés ${ }^{109}$. Les idées de fraternité si chères à la rhétorique révolutionnaire se trouvent ainsi mises en pratique. Avec un événement où « tout le monde se mélange et s'embrasse avec les plus grands signes de joie », s'impose l'idée que les rassemblements de masse ont un rôle à jouer pour unir la communauté française ${ }^{110}$. «La raison triomphe avec le sentiment », proclame encore un autre participant ${ }^{111}$.

(105) Fédération des françois, dans la capitale de l'empire, le 14 juillet 1790: description exacte et détaillée des différentes fêtes, Paris, Porteur des nouvelles, s.d., p. 2.

(106) Samuel STEARN, Dr. Stearn's Tour from Paris to London, Dublin, Sleater and Rice, 1791, p. 33.

(107) Françoise BRUNEL et Sylvain GouJON, Les martyrs de Prairial: textes et documents inédits, Genève, Georg, 1992, p. 258-259.

(108) Nicolas FRÉROT, « La Fédération du 14 juillet 1790 (Souvenir d'un délégué de la ville de Sézanne) », $A H R F$, 8, 1931, p. 164.

(109) Pierre DE VAISSIÈRE, op. cit., p.173.

(110) Françoise BRUNEL et Sylvain GOUJON, op. cit., p. 199.

(111) Le retour du sentiment et de la raison, bouquet à Louis XVI, premier roi citoyen, S.1., Potier de Lille, 1790, 1. 
Comme on l'avait fait à toutes les étapes du mouvement des fédérations, les participants à la fédération nationale du Champ de Mars s'empressent de coucher sur le papier les moments qu'ils viennent de vivre. Ils décrivent leur expérience et tentent de transmettre leurs sensations le plus largement possible. "Il faut, écrit l'auteur de la Confédération nationale, une feuille d'actualité, que je recueille mes sensations pour les déposer dans le sein de mes concitoyens ». On retrouve cette même urgence dans un grand nombre de récits épistolaires de la Fête : «On ne peut s'en faire une idée qu'en la voyant », signale ainsi le Comte de Seneffe, avant de procéder au récit de la cérémonie ${ }^{112}$. "Rien de plus grand, de plus imposant et de plus majestueux », rapporte le représentant d'Avignon dans un courrier à sa ville ${ }^{113}$. Rédigées en l'absence d'un quelconque appareil coercitif contrôlant les lettres ou les publications, ces descriptions démontrent par leur quasi-unanimité, d'une part, le véritable pouvoir d'attraction des fêtes et, d'autre part, à quel point leurs auteurs se sont retrouvés dans une sorte de fusion émotionnelle mêlant expérience vécue et descriptions des fédérations passées ${ }^{114}$.

Les récits des fédérations provinciales du 14 juillet 1790 témoignent de scènes de joie collective similaires à celles vues à Paris. Les responsables municipaux de la ville de Limoges décrivent une fête pensée de façon à permettre la participation la plus large possible ${ }^{115}$. Pour caractériser leur fête, les organisateurs de la fédération du port nord de Saint-Pol évoquent « le spectacle consolant d'un peuple de frères, cherchant mutuellement à se donner les gages de la plus parfaite intimité ». Ils précisent aussi que «chacun a témoigné se trouver heureux du bonheur de tous » ${ }^{116}$. À Bordeaux, "tout, dans ce beau jour, a contribué à manifester avec éclat l'esprit général de civisme qui anime les habitants ${ }^{117}$. Bien que le nombre de récits dont nous disposons soit cette fois limité par rapport aux témoignages rédigés à l'occasion des premières fédérations, signalant peut-être une légère perte d'intérêt dans les régions voyant l'événement pour une seconde fois, les descriptions demeurent très largement positives.

(112) Pierre DE VAISSIÈRE, op.cit.

(113) AM Avignon, 4 H 1 11, 16 juillet 1790.

(114) Un nombre limité d'observateurs, généralement nobles, ont accablé les cérémonies d'injures en privé. Voir Félix de FRANCE D'HEZECQUES, Souvenirs d'un page à la cour de Louis XVI, Paris, Didier, 1873, p.164.

(115) AM Limoges, 2 D 2. Becquart, 1887.

(116) La Fédération du 14 juillet 1790 à Saint-Pol. Procès-verbal de la Fête, St.-Pol,

(117) AM Bordeaux, D 86136. 
À Paris, les célébrations festives se prolongent pendant une semaine. Après la cérémonie principale, les participants se dispersent dans toute une série de banquets organisés localement par les soixante bataillons parisiens de la Garde nationale en l'honneur de leurs visiteurs de province. Une source évoque des tablées prêtes à accueillir 1500 convives dans les couloirs et les jardins de Saint-Martin-des-Champs pour la fête d'une section. D'autres sections dressent de vastes pavillons à la périphérie de la ville, entourant le Champ de la fédération et s'étendant jusqu'aux Champs-Élysées, avec danses et feux d'artifices ${ }^{118}$. Trente mille personnes y dînent en même temps, et avec le « vin et les provisions » distribués à une centaine de milliers de personnes supplémentaires, « la nation se rassembla à la même table ${ }^{119}$. Ce spectacle se répète toutes les nuits pendant plus d'une semaine : alors que les premières festivités ont commencé le 10 juillet, les banquets et les différentes fêtes se poursuivent jusqu'au départ des soldats fédérés le 20 juillet ${ }^{120}$. Dans l'esprit des fêtes civiques de la Rome antique, ces « noces de la liberté », comme le note une brochure, permettent aux populations de s'extraire de leur rythme quotidien pour vivre un moment exceptionnel ${ }^{121}$. La participation ne diminue pas au fil des jours et les dernières célébrations sont tout aussi populaires que les premières. On rapporte que la revue de la Garde nationale parisienne au Champ de Mars le 18 juillet, à l'occasion de laquelle tous les régiments sont rassemblés ensemble en un même lieu pour la première fois, attira « autant de spectateurs que la Fédération ${ }^{122}$.

Et pourtant, la ferveur et l'unanimité apparentes portées par le mouvement de la Fédération ne durent pas. L'été est marqué par les résistances à la Révolution. En août 1790, les contre-révolutionnaires du Vivarais - une région qui avait pourtant joué un rôle central au début du mouvement des fédérations - organisent une démonstration de force au camp de Jalès qui attire cinquante mille personnes. Au nom de la protection de la religion catholique et dans le but de prévenir la résurgence de violences religieuses comme à Nîmes au mois de juillet, l'événement

(118) Fête nationale qui sera célébrée le 15 juillet 1790, Paris, 1790.

(119) Samuel StEARn, op. cit., p. 33 ; Marie-Louise BIVER, Fêtes révolutionnaires à Paris, Paris, PUF, 1979, p. 28. Un autre banquet, auquel participe la délégation avignonnaise, est prévu pour accueillir 15000 convives disposés autour de 150 tables dans la cour du Château de la Muette, AM Avignon, 4 H 111.

(120) Charles-Albert COSTA DE BEAUREGARD, Le Roman d'un royaliste sous la Révolution. Souvenirs du Comte de Virieu, Paris, 1892, p. 200.

(121) Fête nationale, p. 3.

(122) Charles-Albert Costa DE BeAuregard, op. cit., p. 208. Voir également Détail de tout ce qui s'est passé ce matin au Champ-de-Mars, et Discours prononcé par M. de Lafayette, après avoir passé en Revue la Garde-Nationale Parisienne, en présence des Députés \& Volontaires des Gardes-Nationales du Royaume, Paris, L.L. Girard, s.d. 
prend ouvertement la défense de la monarchie contre « les usurpations de la soi-disant Assemblée nationale ${ }^{123}$. Les grands rassemblements qui misent les émotions collectives conviennent aussi bien aux causes conservatrices cherchant à rallier les masses populaires qu'aux projets révolutionnaires. Les fédérations révolutionnaires du 14 juillet 1791 et 1792, à la différence de l'unanimité exprimée lors des fêtes de 1789-1790, sont marquées par des conflits internes - la première célébration prend place au milieu de l'agitation des adversaires de la monarchie à la suite de la fuite du roi à Varennes et la seconde au milieu des tensions qui conduisent au 10 août $1792^{124}$. Jamais ces fêtes ne retrouvent la ferveur émotionnelle qu'elles avaient suscitée en 1789-1790. Si de nombreux rassemblements politiques au cours des $\mathrm{XIX}^{\mathrm{e}}$ et $\mathrm{XX}^{\mathrm{e}}$ siècles s'en sont inspirés, rares sont ceux qui ont su mobiliser comme les fédérations étaient parvenues à le faire et encore plus rares sont ceux qui ont réussi à atteindre la même intensité émotionnelle.

Mouvement concerté qui touche toutes les régions françaises de l'été 1789 à l'été 1790, les fédérations sont le témoignage éloquent de la force émotionnelle engendrée par les débuts de la Révolution. Au-delà de leurs motivations initiales, allant de la défense mutuelle à la citoyenneté ouverte et à l'égalitarisme, les révolutionnaires des petites villes ont inventé une forme radicalement nouvelle d'engagement populaire qui a rapidement fait des émules dans tout le pays en incorporant un nombre toujours plus grand de participants. Pour de nombreux révolutionnaires, les cérémonies ou les fêtes fédératives sont devenues indispensables : elles ont fourni aux élites locales une méthode grâce à laquelle il a été possible de consolider le soutien populaire à leur action, d'affirmer leur position de leader, de témoigner de la force de la garde nationale, d'inclure explicitement des populations plus larges, et par leurs dernières manifestations, de montrer leur puissance et atteindre les autorités départementales puis nationales. De la même façon, elles témoignent du pouvoir de la presse révolutionnaire et de son rôle dans la diffusion des pratiques révolutionnaires. Aucun groupe

(123) Manifeste et protestation de cinquante mille Français fidèles, armés dans le Vivarais, pour la cause de la Réligion et de la Monarchie. Contre les usurpations de l'Assemblée se disant Nationale, S.1., s.d ; Charles Jolivet, La Révolution dans l'Ardèche, Largentière, Mazel, 1930, p. 209-21.

(124) Sur les tensions au cours des fédérations parisiennes, voir, pour 1791, Journal des Clubs, 23 juillet 1791 et la pétition des Cordeliers datée du 14 juillet, AN C 75 737. Pour 1792, voir Alessandro FOnTANA, Francesco FURLAN et Georges SARO (dir.), Venise et la Révolution française: les 470 dépêches des ambassadeurs de Venise au Doge, 1786-1795, Paris, Lafont, 1997, p. 765 ; Charles-Albert COSTA DE BEAUREGARD, p. 360. 
ne semble avoir été contraint à participer : la dynamique qui s'est créée pour former ce mouvement d'envergure nationale est la caractéristique principale des fédérations. Le projet révolutionnaire, et l'identification avec une nation française commune, s'est imposé dans les esprits à travers la participation collective.

Plus largement, on ne peut pas comprendre la Révolution française si l'on ne s'efforce pas d'intégrer à son raisonnement la vive émotion qu'elle provoqua chez les contemporains. À travers l'exercice de l'affirmation collective, la force de la Révolution - et de ses participants - s'est affermie. Les fédérations se sont imposées à la fois comme moyen de se libérer des peurs caractéristiques de la période, et comme espace de l'affirmation collective des espoirs des participants. Dans une période de mobilisation populaire croissante, les fédérations ont fourni un modèle, un cadre pour donner à voir la force de l'action collective : la libération collective d'émotions a contribué à faire l'unité des participants et à créer une nouvelle société.

Micah AlPAUGH University of Pennsylvania / University of Central Missouri

Wood Building, Room 136

Warrensburg, MO 64093

micahalpaugh@gmail.com

Traduction assurée par Julien Louvrier 